\title{
Application of Fourier Series for Evaluation of Roundness Profiles in Metrology
}

\author{
Augustín Görög', Ingrid Görögová1* \\ 1 Slovak University of Technology in Bratislava, Faculty of Materials Science and Technology in Trnava, J. Bottu \\ 25, 91724 Trnava, Slovak Republic \\ * Corresponding author's e-mail: ingrid.gorogova@stuba.sk
}

\begin{abstract}
A Fourier series is an expansion of a periodic function $\mathrm{f}(\mathrm{x})$ in terms of an infinite sum of sines and cosines. Fourier series make use of the orthogonality relationships of the sine and cosine functions. The computation and study of Fourier series are known as harmonic analysis. It is a useful way to break up an arbitrary periodic function into a set of simple terms that can be plugged in, solved individually, and then recombined to obtain the solution to the original problem or an approximation to it to whatever accuracy is desired or practical. This paper deals with the mathematical basics of Fourier series using trigonometric functions. This is the basic for a discrete Fourier transform. It allows transforming the discrete data to the frequency data or vice versa, i.e. transforming the frequency data to the discrete data. The most important part of the article is the application of the Fourier series and the Fourier transform to metrology, specifically on the roundness profile. The mathematical relationships for the practical use of harmonic analysis and the detailed method of determining the actual phase were described. General relationships do not give accurate results, due to the phase shift quadrant. The results of the harmonic analysis were applied graphically by the authors on a concrete example of a roundness profile. The individual harmonic components are shown in the linear and polar graphs as well as the resulting roundness profile. The Fourier analysis knowledge will contribute to a better analysis of the roundness profiles measured on the drawn tubes that will be investigated in the research project.
\end{abstract}

Keywords: Fourier series; harmonic analysis; roundness profile; actual phase.

\section{INTRODUCTION}

Transmission and processing the data is most commonly implemented in the form of electrical signals expressed the time-variable physical quantities, if necessary, its spectrum. The signal is characterized by a set of harmonic amplitudes of the various frequencies, and the set of their initial phases (amplitude and phase spectrum). The spectral analysis of waveforms is called spectral (harmonic) analysis [6]. The harmonic analysis is the foundation for much of modern mathematical analysis. On the basis of the idea of breaking up an arbitrary function into simple component functions, the harmonic analysis has become a powerful and pervasive part of mathematics. Image processing, signal compression, partial differential equations, control theory, and many other essential parts of engineering and applied mathematics are studied using the aspects of harmonic analysis. Fourier series, Fourier transforms, pseudodifferential operators, and wavelets are all aspects of the subject that find application in the real world problems [11]. Clue [4] describes a method of calculating the transforms, currently obtained via Fourier and reverse Fourier transforms.

The method allows efficiently calculating the transforms of a signal having an arbitrary dimension of the digital representation by reducing the transform to a vector-to-circulant matrix multiplying. Bandara [3] showcases a number of non-trivial geometric problems that have recently been resolved by the marrying methods from functional calculus and real-variable harmonic 
analysis. He gives a brief description of these methods, as well as their interplay. His survey was succinct, rather than comprehensive, and its aim was to inspire geometers and analysts alike to study these methods so that they can be adapted and potentially applied more widely. Pérez de Viñaspre [14] presents the evidence on the validity of the application of the spectral analysis to the economic phenomena in his paper. Karateev et al. [8] reviewed some aspects of harmonic analysis for the Euclidean conformal group, including conformally-invariant pairings, the Plancherel measure, and the shadow transform. They introduced two efficient methods for computing these quantities: one based on weight-shifting operators, and another based on Fourier space. Viitala et al. [21] analyzed the uncertainty of the four-point roundness measurement of a bearing inner ring using harmonic components of the Fourier series. Attention was drawn especially to the phase of the harmonic components, since the uncertainty of the phase is analyzed very little in the existing research studies. Adamczak et al. [1, 2] compared scratch or other significant disturbance of the profile with Fourier and Wavelet Transform. They concluded that both types of transforms can complement each other when evaluating geometrical surface structures.

A lot of other authors [7, 12, 17, 16,9] deal with the applications of harmonic analysis in the areas as diverse as number theory, representation theory, signal processing, quantum mechanics, tidal analysis and metrology.

\section{FOURIER SERIES}

The periodic function [15] on the numerical axis " $x$ " is a function that the functional value does not change, when added to the " $T$ " period:

$$
f(x+T)=f(x)
$$

The general periodic function can be decomposed into "simple periodic functions" - "simple harmonic functions", meaning functions of the type:

$$
f(x)=c \sin (n x+\gamma)
$$

With periods:

$$
T=\frac{2 \pi}{n}
$$

Where: $c$ - the amplitude of harmonic function, $n$ - the frequency of harmonic function, $\gamma-$ the phase shift of harmonic function.
After adjustment:

$$
\begin{aligned}
& f(x)=c(\sin (n x) \cos \gamma+\cos (n x) \sin \gamma) \\
& f(x)=c \sin (n x) \cos \gamma+c \cos (n x) \sin \gamma)
\end{aligned}
$$

If we define constant:

$$
\begin{aligned}
& a=c \cos \gamma \\
& b=c \sin \gamma
\end{aligned}
$$

We receive:

$$
f(x)=a \cos (n x)+b \sin (n x)
$$

When dividing the function with the $2 \pi$ period to simple harmonic functions, it is necessary to select the frequencies of each harmonic function so that each harmonic function had a value of $2 \pi$ as one of its periods. This means that each harmonic component must be taken as harmonic functions with entire frequencies (frequencies " $n$ " are integer values $n \in(1,2, \ldots, \infty))$.

Universal periodic function $f(x)$ with period $2 \pi$ can be decomposed into a series of the form:

$$
\begin{gathered}
f(x)=a_{0}+\left(a_{1} \cos x+b_{1} \sin x\right)+ \\
+\left(a_{2} \cos (2 x)+b_{2} \sin (2 x)\right)+\cdots+ \\
+\left(a_{n} \cos (n x)+b_{n} \sin (n x)\right)+\cdots \\
\text { Or simplified: } \\
\quad f(x)=a_{0}+\sum_{n=1}^{\infty}\left(a_{n} \cos (n x)+\right. \\
\left.\quad+b_{n} \sin (n x)\right)
\end{gathered}
$$

This series is called "Fourier series" and the constants $a_{0}, a_{1}, a_{2}, \ldots a_{n}, b_{1}, b_{2}, \ldots b_{n}$ are called Fourier coefficients.

Fourier coefficients can be calculated by the Euler formulas [22]:

$$
\begin{gathered}
a_{0}=\frac{1}{T} \int_{0}^{T} f(x) d x \\
a_{n}=\frac{2}{T} \int_{0}^{T} f(x) \cos (n \omega x) d x \\
b_{n}=\frac{2}{T} \int_{0}^{T} f(x) \sin (n \omega x) d x
\end{gathered}
$$

\section{DISCRETE FOURIER TRANSFORM - DFT}

The discrete Fourier transform [18] changes an $N$ point input signal into two $N / 2+1$ point output signals. The input signal contains the signal being decomposed. The two output signals contain the amplitudes of the component sine and cosine waves. The most common type of signal entering the DFT is composed of the samples taken at regular intervals of time (it might be a general reference to any discrete signal that is being decomposed). Because the input signal is said to be 
in the time domain, the term frequency domain is used to describe the amplitudes of the sine and cosine waves (Fig. 1). In the time domain, $x[$ ] consists of the $N$ points running from 0 to $N-1$. In the frequency domain, the DFT produces two signals: 1. the real part - written $\operatorname{ReX}[]$,

2. the imaginary part - written $\operatorname{ImX}[]$.

Each of these frequency domain signals run from 0 to $N / 2$ (are $N / 2+1$ points long). The Forward DFT transforms from the time domain to the frequency domain. The Inverse DFT transforms from the frequency domain to the time domain.

\section{APPLICATION OF THE FOURIER SERIES TO A ROUNDNESS PROFILE}

The roundness profile obtained by measuring roundness can be represented both polarly and linearly (Fig. 2). The tolerance zone in the considered cross-section is limited by two concentric circles with a difference in radii of " $t$ " [5]. The roundness value from the roundness profile is determined by one of four methods $[20,13]$ :

- Minimum Zone circle (MZC),

- Least Squares Circles (LSC),

- Maximum Inscribed Circle (MIC),
- Minimum Circumscribed Circle (MCC).

The measured and evaluated value was compared with the nominal value of tolerance which was prescribed in the drawing documentation. The measured value must be less than or equal to the prescribed value. If it exceeds this value, the measured roundness does not meet the required values and therefore the produced area is incorrect.

The roundness profile is a periodic function, and therefore it is possible to apply knowledge of periodic functions and Fourier series to this profile as well. The roundness profile is measured in polar coordinates and therefore the radius " $r$ " is a function of the angle " $\gamma$ ":

$$
r=f(\gamma)
$$

This function is repeated with the $2 \pi$ period:

$$
r(\gamma)=r(\gamma+2 k \pi)
$$

where: $k= \pm 1, \pm 2, \ldots, \pm \infty$

It is possible to use the Fourier series for the mathematical expression of the roundness profile:

$$
\begin{gathered}
r(\gamma)=r_{0}+c_{1} \cos \left(\gamma-\gamma_{1}\right)+ \\
+c_{2} \cos \left(2 \gamma-\gamma_{2}\right)+\cdots+ \\
+c_{n} \cos \left(n \gamma-\gamma_{n}\right)+\cdots
\end{gathered}
$$

or by the summation sign

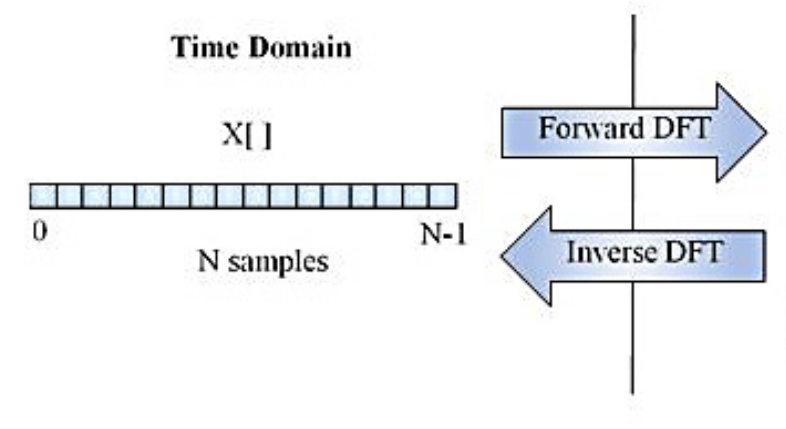

Frequency Domain

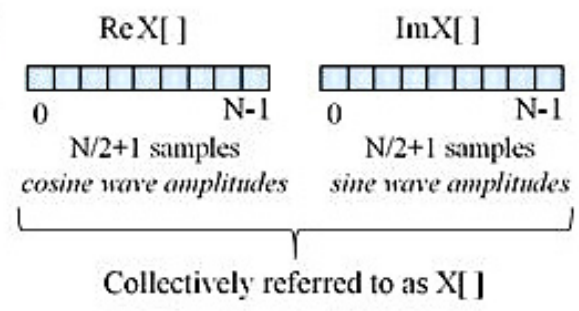

Fig. 1. The discrete Fourier transform [18]
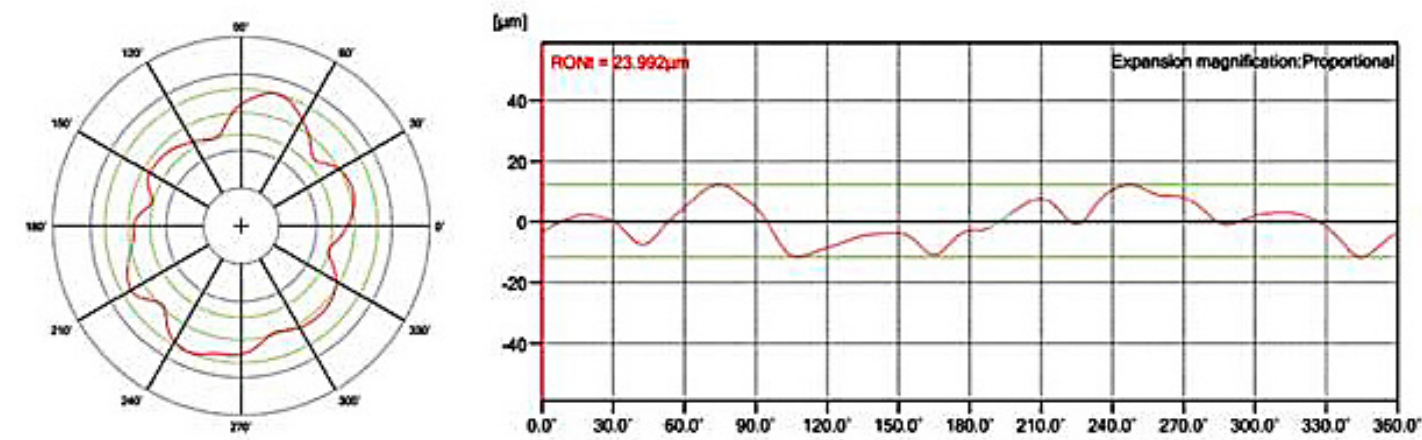

Fig. 2. Round profile - polar and linear view 


$$
r(\gamma)=r_{0}+\sum_{n=1}^{\infty} c_{n} \cos \left(n \gamma-\gamma_{n}\right)
$$

where: $c_{n}$ - the amplitude of the n-th harmonic function, $n$ - the frequency of the $n$-th harmonic function, $\gamma_{n}-$ the phase shift of the $n$-th harmonic functions.

The above-mentioned forms of Fourier series are not preferred because they contain initial phase $\gamma_{n}[6]$. Therefore, by gradual modification can be formula write in the form:

$$
\left.r(\gamma)=r_{0}+\sum_{n=1}^{\infty} a_{n} \cos (n \gamma)+b_{n} \sin (n \gamma)\right)
$$

Instead of original coefficients $\gamma_{n}$ determining the $\mathrm{n}$-th harmonic, we obtain the new coefficients $a_{n}$ and $b_{n}$.

$$
a_{n}=c_{n} \cos \gamma_{n}
$$

$$
b_{n}=c_{n} \sin \gamma_{n}
$$

The following applies for amplitude:

$$
c_{n}=\sqrt{a_{n}^{2}+b_{n}^{2}}
$$

And for phase:

$$
\gamma_{n}=\operatorname{arctg} \frac{b_{n}}{a_{n}}
$$

Table 1 presents the difference between the calculated $\left(\gamma_{c}\right)$ and the actual $(\gamma)$ phases - the actual phase depends on whether the values " $a$ " and " $b$ " are positive $(+)$ or negative (-). The table is supplemented by examples and graphical interpretation.

The roundness profile was measured on roundness measuring machine discreetly - the profile forms several discrete points. It is possible to obtain Fourier coefficients using the discrete Fourier transform (DFT) from these points:

Table 1. Calculated and actual phase

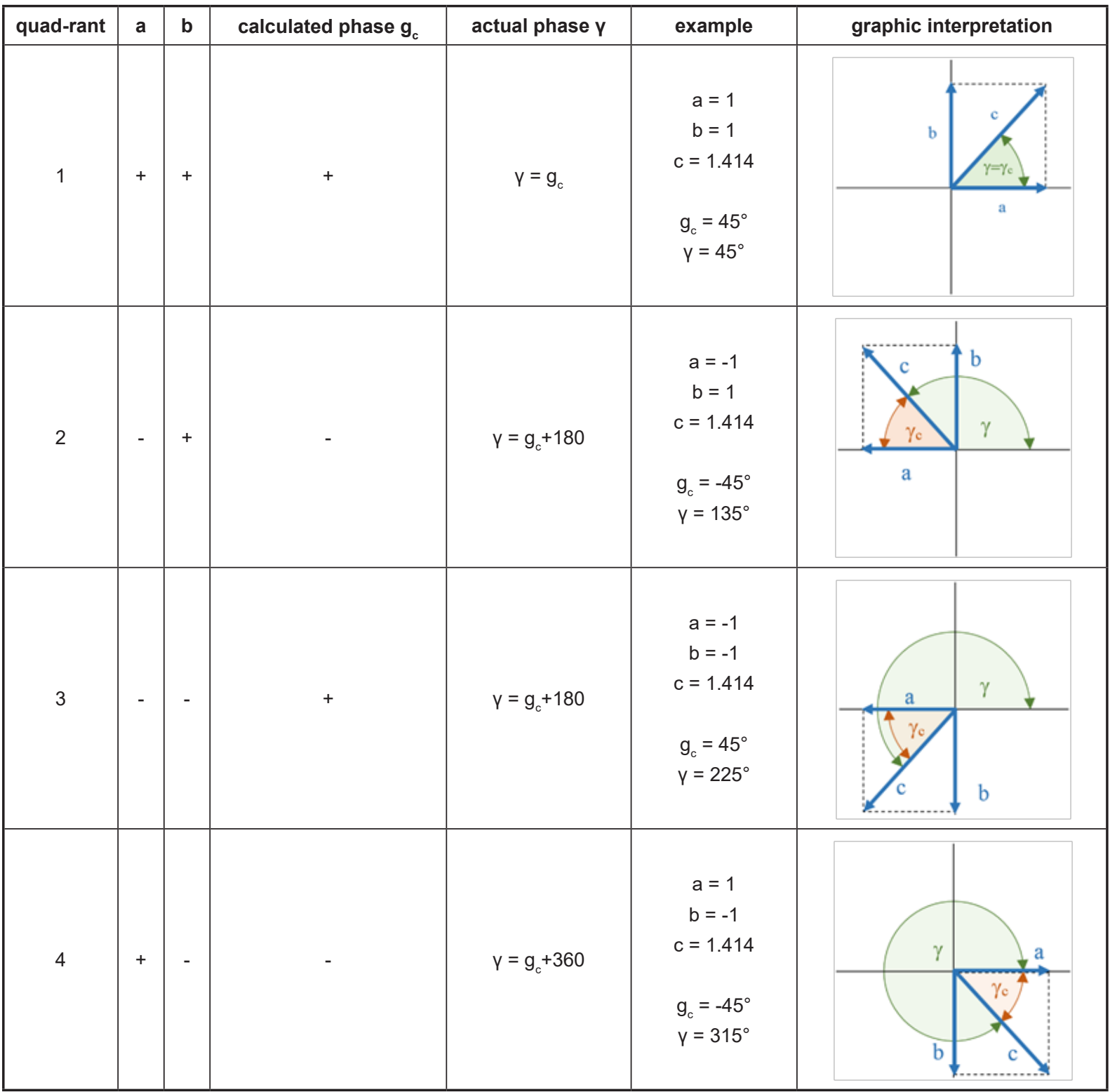




$$
\begin{aligned}
& a_{n}=\frac{2}{N} \sum_{p=1}^{N} r_{p} \cos \frac{2 \pi n p}{N} \\
& b_{n}=\frac{2}{N} \sum_{p=1}^{N} r_{p} \sin \frac{2 \pi n p}{N}
\end{aligned}
$$

where: $N$ - number of discreete points, $r_{p}$ - coordinate (radius) $\mathrm{p}$-th point, $n-$ frequency of the $n$-th harmonic function, $p$ - serial number of points.

\section{HARMONIC ANALYSIS OF THE ROUND PROFILE}

The roundness measuring machine typically provides the ability to analyze the round profile in terms of Fourier analysis (sometimes called harmonic analysis). The software will write amplitudes $(c)$ and phases $(\gamma)$ for a given round profile. Each harmonic component has its amplitude $\left(c_{1}\right.$, $\left.c_{2}, c_{3}, \ldots\right)$ and phase $\left(\gamma_{1}, \gamma_{2}, \gamma_{3}, \ldots\right)$. The software does not write Fourier coefficients $(a, b)$ that can be calculated from amplitudes and phases. Tab. 2 presents the amplitude $\left(c_{n}\right)$ and phase $\left(\gamma_{n}\right)$ values from a particular roundness measurement. The roudness was measured (3600 points) at RONDCOM 60A (Accretech), The Fourier coefficients $\left(a_{n}, b_{n}\right)$ are calculated from these values.

The individual harmonic components (which are defined by the amplitudes and phases in Table 2 ) are shown graphically in Table 3 . They are displayed in a linear and polar graph. For comparison, all linear graphs are on the same scale as well as all polar graphs on the same scale. For better il- lustration, the linear graphs do not have the same scale as the polar graphs.

The roundness profile was created by adding individual harmonic components. Thus, it is the sum of all 15 harmonic components (roundness profile $\left.=\mathrm{HC}^{1 \mathrm{st}}+\mathrm{HC}^{2 \mathrm{nd}}+\ldots+\mathrm{HC}^{15 \text { th }}\right)$. This profile is shown graphically in Table 4. It was necessary to change the display scale in comparison with the previous graphs of harmonic components.

\section{CONCLUSIONS}

The harmonic analysis can be used to decompose measured profiles into individual harmonic components in metrology. Fourier series are used for this analysis. On the basis of the harmonic analysis, it is possible to analyze e.g. the influence of technological production conditions on the geometric accuracy of the produced areas or to determine the causes of geometric inaccuracies (deviations). As a result, deviations can be avoided, and production quality can be improved. The Fourier analysis knowledge will contribute to a better analysis of the roundness profiles measured on the drawn tubes that will be investigated in the research project. This will enable to better compare the roundness profile before and after drawing the tubes and hence analyze the shape change of the roundness profile in the tubes forming process more accurately. Therefore, it is necessary to know the mathematical basics of harmonic analysis and apply it to the measured profiles. The har-

Table 2. The values of amplitude, phase, and calculated the Fourier coefficients

\begin{tabular}{|c|c|c|c|c|}
\hline Harmonic component $(n)$ & Amplitude $\left(\mathbf{c}_{n}\right)[\mu \mathrm{m}]$ & Phase $\left(\mathbf{V}_{\mathbf{n}}\right)\left[^{\circ}\right]$ & $\mathbf{a}_{\mathrm{n}}=\mathbf{c}_{\mathbf{n}} \cos \mathbf{Y}_{\mathrm{n}}$ & $\mathbf{b}_{\mathrm{n}}=\mathbf{c}_{\mathrm{n}} \sin \mathbf{Y}_{\mathrm{n}}$ \\
\hline $1^{\text {st }}$ & 6.70835 & 270.927 & 0.10853 & -6.70747 \\
\hline $2^{\text {nd }}$ & 12.23454 & 65.518 & 5.07008 & 11.13455 \\
\hline $3^{\text {rd }}$ & 2.90168 & 70.151 & 0.98524 & 2.72929 \\
\hline $4^{\text {th }}$ & 2.88858 & 72.120 & 0.88686 & 2.74907 \\
\hline $5^{\text {th }}$ & 3.88372 & 7.812 & 3.84768 & 0.52789 \\
\hline $6^{\text {th }}$ & 10.09228 & 16.564 & 9.67347 & 2.87717 \\
\hline $7^{\text {th }}$ & 0.93399 & 17.745 & 0.88955 & 0.28466 \\
\hline $8^{\text {th }}$ & 0.88487 & 21.082 & 0.82564 & 0.31829 \\
\hline $9^{\text {th }}$ & 1.10167 & 4.377 & 1.09846 & 0.08408 \\
\hline $10^{\text {th }}$ & 1.18360 & 28.134 & 1.04375 & 0.55811 \\
\hline $11^{\text {th }}$ & 1.06584 & 14.492 & 1.03193 & 0.26672 \\
\hline $12^{\text {th }}$ & 2.85383 & 29.811 & 2.47618 & 1.41875 \\
\hline $13^{\text {th }}$ & 0.49441 & 19.393 & 0.46636 & 0.16417 \\
\hline $14^{\text {th }}$ & 0.36566 & 24.203 & 0.33352 & 0.14991 \\
\hline $15^{\text {th }}$ & 0.09695 & 3.496 & 0.09677 & 0.00591 \\
\hline
\end{tabular}


Table 3. Linear and polar graphs of individual harmonic components (HC)

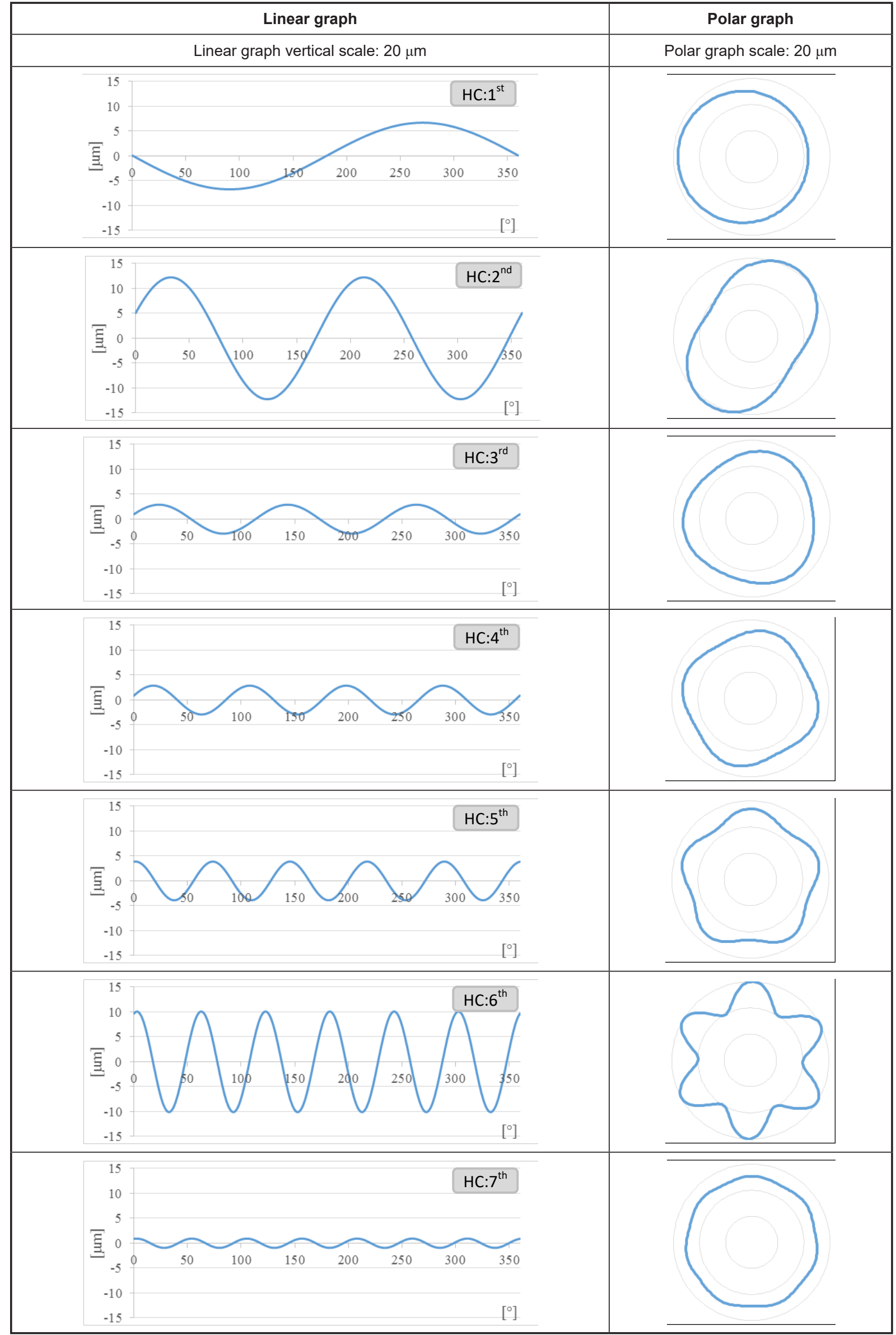




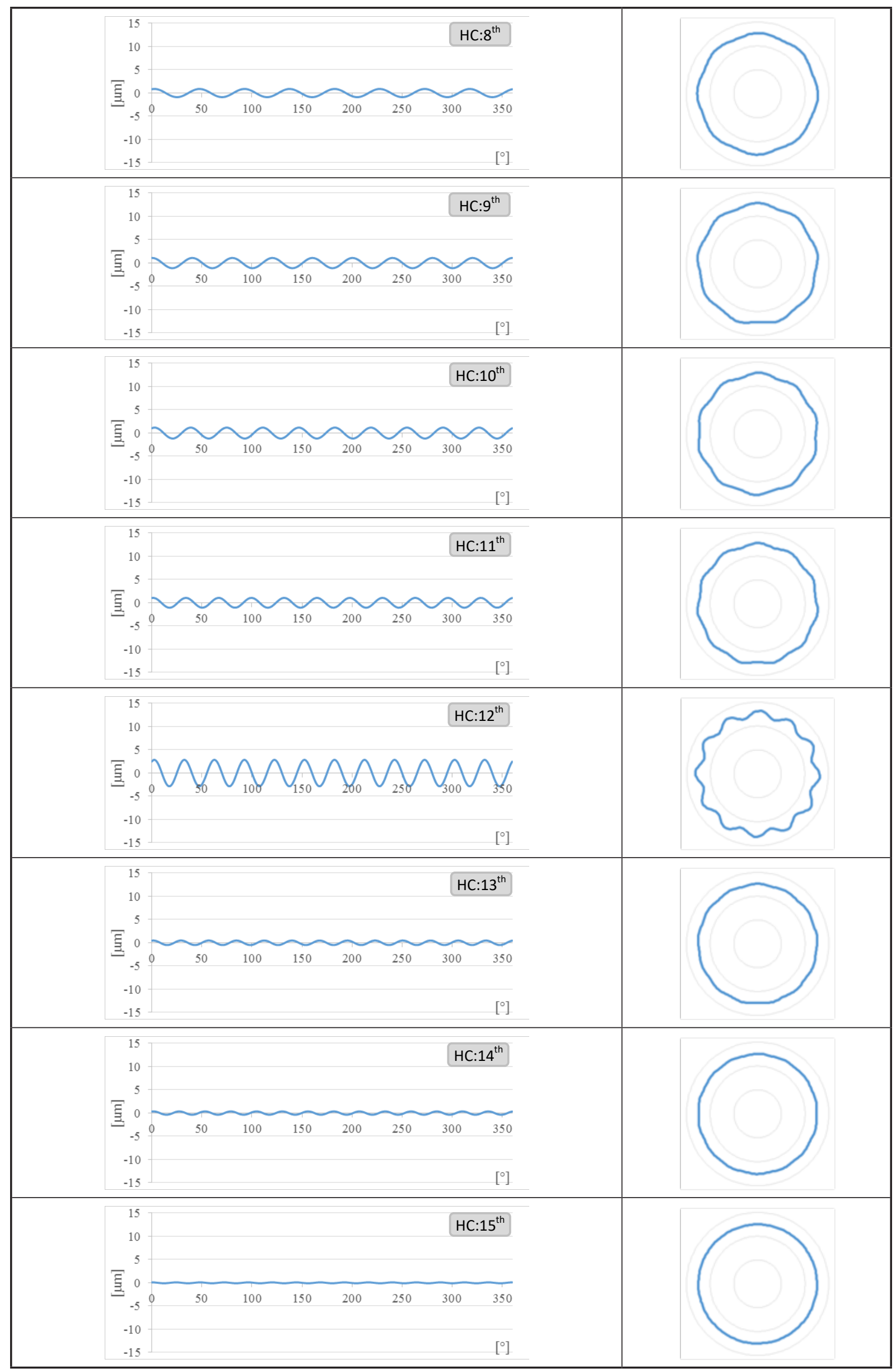


Table 4. Linear and polar graph of roundness profile (harmonic components 1 st to 15 th)

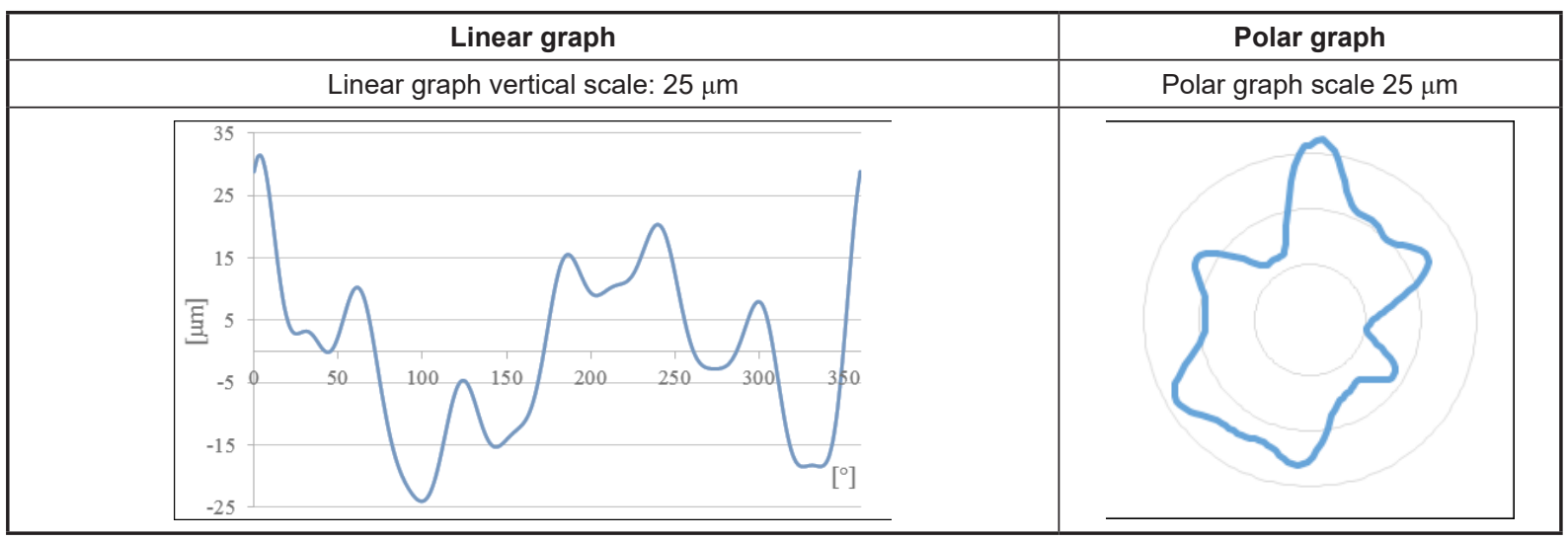

monic analysis can be applied in the area of surface macrogeometry (geometric tolerances) but also in the area of surface microgeometry (surface roughness $[10,19])$. The article focused on applying the harmonic analysis to a roughness profile that belongs to the area of geometric tolerances. The use of analysis on other profiles (e.g. straightness or surface roughness) would be similar.

\section{Acknowledgement}

This contribution was supported by the VEGA Grant No. 1/0122/16 Research of deformation processes using spatial reconstruction of microstructure and shape of formed parts. This support is gratefully acknowledged.

\section{REFERENCES}

1. Adamczak, S., Makieła, W., Stepien, K. Investigating Advantages and Disadvantages of the Anaysis of a Geometrical Surface Structure with the Use of Fourier and Wavelet Transform. Metrology and Meaurement Systems 17, 2010, DOI 10.2478/ v10178-010-0020-x.

2. Adamczak, S., Makieła, W., Stepien, K. Fourier Transform vs. wavelet transform in analysis of geometrical surface structure. 10th International Scientific Conference - New Ways in Manufacturing Technologies, 77-84, 2010.

3. Bandara, L. Functional calculus and harmonic analysis in geometry. https://arxiv.org/abs/1812.11795, 2018.

4. Clue, V. Harmonic analysis. Proceedings of the 2004 IEEE Electro/Information Technology Conference. Milwaukee USA, 2004, 53-58.

5. Görög, A., Görögová, I. Current concept of geometrical accuracy. Research papers Faculty of Materials Science and Technology Slovak Univer- sity of Technology in Trnava, 22(34), 2014, 43-50, ISSN 1336-1589.

6. Görögová, I. Technological Heredity in Bearing Production. Plzeň: Aleš Čeněk, ISBN 978-807380-599-9, 2016.

7. Grafakos, L., Oliveira, S.D., Pramanik, M., Seeger, A., Stovall, B. Some Problems in Harmonic Analysis. https://arxiv.org/pdf/1701.06637.pdf, 2017.

8. Karateev, D., Kravchuk, P., Simmons-Duffin, D. Harmonic Analysis and Mean Field Theory. https:// arxiv.org/abs/1809.05111, 2018.

9. Kocsis, P., Balla, P., Antal, Á. Wavelet-based Optimization of Surface Reconstruction. Acta Polytechnica Hungarica, 15 (4), 179-198, ISSN 17858860, DOI: 10.12700/APH.15.4.2018.4.1, 2018.

10. Kowalski, M. Wiśniewska, M. Karolczak, P. Stančeková, D. Applicability of selected groups of roughness parameters for description of surface layer of flat - top "plateau" structures. Advances in science and technology research journal, Vol.: 10 Issue: 32, 2016 Pages: 32-39, ISSN 2299-8624.

11. Krantz, S.G. Harmonic analysis. WIREs Comp Stat 3, 163-167, DOI:10.1002/wics.143, 2011.

12. Li, J. Harmonic analysis of stationary measures. General Mathematics [math.GM]. Université deBordeaux, https://tel.archives-ouvertes.fr/tel01975306/document, 2018.

13. Némedi, I., Sekulić, M., Radlovački, V., Hodolič, J., Hadžistević, M., Takács, M. Method for Determining Roundness and Actual Form of Circular Workpiece Cross Sections, Acta Polytechnica Hungarica, 14(6), 2017, 169-184, ISSN 1785-8860 DOI: 10.12700/APH.14.6.2017.6.10.

14. Pérez de Viñaspre, F.P., Pérez Pascual, P.A. Harmonic Analysis: The Aplication of 'Theoretical Cycles' to the Economic Analysis. Economic cycles, 2 (1), 2001.

15. Romanovskij, P.I. Fourier series. Field theory. Analytical and special functions. Laplace transform. Praha, SNTL, 1964. 
16. Sankararaman, S., Varun, V.S., Balakrishnan, A., Devi, H.V. Harmonic analysis of rectifier and amplifier outputs. 4th International Seminar of Swamy Vivekananda Association of Science and Humanities (SVASH) At: Vyloppilli Samskrithi Bhavan, Trivandrum, Kerala, 2018.

17. Sawano, Y. Elementary Facts on Harmonic Analysis. Theory of Besov Spaces, Developments in Mathematics 56, DOI: 10.1007/978-981-13-08369_1, 2018.

18. Smith, S.W. The Scientist and Engineer's Guide to Digital Signal Processing, California Technical Publishing, San Diego, California, ISBN 0-9660176-6-8, 1999.

19. Stancekova, D. Mrazik, J. Rybicka, I. Naprstkova,
N. Kraus, P. An Impact of Technological Conditions on Surface Burning in Grinding of the Orbit. Advances In Science And Technology, 12 (4), 2018, 19-27, ISSN 2299-8624.

20.Sui, W., Zhang, D. Four Methods for Roundness Evaluation. Physics Procedia, Vol. 24, part C, 2012, 2159-2164. DOI: 10.1016/j.phpro. 2012.02.317.

21. Viitala, R., Widmaier, T., Hemming, B., Tammi, K., Kuosmanen, P. Uncertainty analysis of phase and amplitude of harmonic components of bearing inner ring four-point roundness measurement. Precis. Eng., vol. 54, 2018, 118-130.

22. Walther, E. et al. Technical formulas. Bratislava, Alfa, 1984. 